\title{
Matrix theory origins of non-geometric fluxes
}

\author{
Athanasios Chatzistavrakidis ${ }^{1}$ and Larisa Jonke ${ }^{1,2}$ \\ ${ }^{1}$ Bethe Center for Theoretical Physics and Physikalisches Institut, University of Bonn, \\ Nussallee 12, D-53115 Bonn, Germany \\ ${ }^{2}$ Theoretical Physics Division, Rudjer Bošković Institute, \\ Bijenička 54, 10000 Zagreb, Croatia \\ E-mails: than@th.physik.uni-bonn.de, larisa@irb.hr
}

\begin{abstract}
We explore the origins of non-geometric fluxes within the context of $\mathrm{M}$ theory described as a matrix model. Building upon compactifications of Matrix theory on non-commutative tori and twisted tori, we formulate the conditions which describe compactifications with non-geometric fluxes. These turn out to be related to certain deformations of tori with non-commutative and nonassociative structures on their phase space. Quantization of flux appears as a natural consequence of the framework and leads to the resolution of nonassociativity at the level of the unitary operators. The quantum-mechanical nature of the model bestows an important role on the phase space. In particular, the geometric and non-geometric fluxes exchange their properties when going from position space to momentum space thus providing a duality among the two. Moreover, the operations which connect solutions with different fluxes are described and their relation to T-duality is discussed. Finally, we provide some insights on the effective gauge theories obtained from these matrix compactifications.
\end{abstract}




\section{Contents}

1 Introduction $\quad 1$

2 The matrix model and its compactifications $\quad 4$

2.1 The BFSS matrix model . . . . . . . . . . . . . . . . . 4

2.2 Compactification on tori . . . . . . . . . . . . . . . . . 4

2.3 Compactification on twisted tori . . . . . . . . . . . . . . . . 6

3 Non-geometric compactifications of Matrix theory $\quad 8$

3.1 Algebraic building blocks for fluxes . . . . . . . . . . . . . . . . . 9

3.2 Moving from block to block - T-duality . . . . . . . . . . . . . . . . 15

3.3 Position/Momentum space duality . . . . . . . . . . . . . . . . 17

3.4 Resolution of non-associativity and flux quantization . . . . . . . . . . 21

3.5 Putting the blocks together . . . . . . . . . . . . . . . 22

4 Remarks on the gauge theory 23

4.1 Gauge theory with fluxes . . . . . . . . . . . . . . . . . . . 24

5 Conclusions $\quad 26$

\section{Introduction}

Superstring theories offer an attractive framework for the ultraviolet completion of our current knowledge of nature as described by the standard model of particle physics and general relativity for gravitational interactions. As such, there is hope that superstring theories will ultimately account for physics both at the weak scale and at the Planck scale. A deeper conceptual unification of superstring theories, including the several dualities among themselves, is achieved in the context of $\mathrm{M}$ theory, whose full quantum-mechanical incarnation remains however elusive. A very interesting proposal for its non-perturbative definition was provided in Ref. [1] and is known under the name of Matrix theory.

The attempt to connect superstring theories to our low-energy, four-dimensional world traditionally involves a compactification of the ten-dimensional theory and a subsequent dimensional reduction to four dimensions. Clearly, finding the correct vacuum which would reproduce the standard model at low energies is not an easy task. A lot of attention in the recent years focused on flux compactifications [2,3], where the internal components of p-form fields, present in string theory, acquire a vacuum expectation value. This longstanding programme is mainly carried out in the supergravity approximation, which is the low-energy, field theory limit of perturbative string theory. 
A separate development, mainly in the direction of providing a non-perturbative definition of superstring theories, arose in the context of reduced matrix models [4]. In this framework, the dynamical variables are represented by large Hermitian matrices which provide the microscopical degrees of freedom of superstrings. In the case of the type IIB superstring theory such a model was proposed in Ref. [5] and it was studied further in numerous instances. These range from the study of non-commutative Yang-Mills theories [6], the structure and dimensionality of spacetime [7-9] and the emergence of geometry and gravity [10] to applications in particle physics $[11,12]$ and cosmology $[13,14]$. It is worth mentioning that these studies use both analytical tools and/or Monte Carlo simulations. A recent review of the latter with a more complete list of references is Ref. [15]. Moreover, as already mentioned, the authors of Ref. [1] suggested a matrix model serving as a non-perturbative formulation of $\mathrm{M}$ theory, called Matrix theory.

In connection to the string compactification programme, a natural development was the study of compactifications in the framework of Matrix theory. The first systematic study was performed by Connes, Douglas and Schwarz in Ref. [16], where toroidal compactifications of Matrix theory were examined and important relations to non-commutative geometry and non-commutative gauge theories were described. It was argued that matrix compactifications on non-commutative tori can be related to supergravity compactifications. In particular, a constant deformation of the torus leads to a theory which is tantamount to a vacuum of eleven-dimensional supergravity with constant background three-form potential. In type IIA language, there is a reciprocal relation among the constant non-commutativity parameter $\theta$ and a constant B-field.

The relation between non-commutativity parameters and background values of fields raises the question whether this analogy can be extended to more general situations, i.e. how flux compactifications can be understood in matrix models. Conventional string compactifications may include geometric fluxes and NS-NS fluxes, as well as R-R fluxes in the type II cases [2,3]. A first description of geometric fluxes in Matrix theory was given in Ref. [17] for the case of the three-dimensional twisted torus. This was recently revisited and generalized to higher-dimensional twisted tori, utilizing their construction as quotients of nilpotent Lie groups by certain discrete subgroups of them (nilmanifolds) [18]. However, the possibility of describing NS-NS flux compactifications in this framework has not been studied yet ${ }^{1}$. What is more, a lot of attention was drawn recently to the so-called non-geometric fluxes corresponding to unconventional compactifications whose origin is not yet fully understood. Clearly, their possible role in Matrix theory was not yet addressed.

Non-geometry is intimately connected to T-duality $[20-22]^{2}$. Moreover, it may be related to an unconventional type of fluxes which can be present in the effective superpotential of a string compactification $[26,27]$. A convenient way to think of such backgrounds has as starting point a toroidal compactification of string theory on a standard torus penetrated by a NS-NS flux $H_{i j k}$. As usual, let us restrict our discussion to a three-dimensional torus, keeping in mind that this is not a fully consistent background of string theory and has to be appropriately extended, as discussed in Ref. [28]. It serves as a toy model, whose central properties may be directly transferred to a full-fledged vacuum. Performing a T-duality

\footnotetext{
${ }^{1}$ See however Ref. [19].

${ }^{2}$ For T-duality in Matrix theory, see for example the Refs. [23-25].
} 
along one direction of the torus the T-dual geometry is described by the three-dimensional nilmanifold, the twisted torus, whose non-trivial spin connection serves as a geometric flux. Such backgrounds lie in the heart of Scherk-Schwarz compactifications [29] and they were studied systematically in [30] and more recently in [28,31-34]. A second T-duality along another direction of the torus takes the twisted torus background to a situation which is globally ill-defined. The fields of the theory cannot be patched with the usual transition functions anymore; instead this patching requires T-duality elements [35]. The situation gets even worse when a third T-duality is considered. This bizarre situation was tackled in the context of the doubled formalism, where an extended space is considered such that duality transition functions become diffeomorphisms of the enlarged manifold [35]. Further progress led to the construction of twisted doubled tori, which provided a context where both geometric and non-geometric situations can be described [36,37]. The above connection between NS-NS flux $(H)$, geometric flux $(f)$ and non-geometric fluxes $(Q$ and $R$ respectively) may be described by the following T-duality chain:

$$
H_{i j k} \stackrel{T_{k}}{\longrightarrow} f_{i j}{ }^{k} \stackrel{T_{j}}{\longrightarrow} Q_{i}^{j k} \stackrel{T_{i}}{\longrightarrow} R^{i j k} .
$$

More recently, a ten-dimensional description of non-geometric fluxes was investigated in the context of generalized geometry [38,39], double field theory [40-43] and non-associative geometry $[44,45]^{3}$.

From the early studies of non-geometric backgrounds it was realized that $Q$ fluxes are somehow associated to non-commutativity and $R$ fluxes to non-associativity of the underlying space [47-50]. Recently, the emergence of non-commutative and non-associative geometries in compactifications with non-geometric fluxes was described by Lüst from a physically motivated perspective $[51,52]$. In particular, it can be related to the properties of a quantum-mechanical particle moving under the influence of a (non-constant) magnetic field $[53,54]$. This is also reminiscent of the Landau problem in quantum mechanics (see for example the discussion in Ref. [55]). Furthermore, the quantization of such backgrounds was recently elaborated in Ref. [56]. Such structures were also derived using conformal field theory in Ref. [57] and later also appeared in the context of asymmetric orbifolds [58].

Having already mentioned the close connection between non-commutativity and compactifications of Matrix theory, it is worth examining whether non-geometric fluxes can be traced in non-commutative and/or non-associative deformations of tori in this context. This is the main topic of the present paper. We believe that there are some advantages in this programme as compared to investigations of such structures directly in supergravity. First of all, Matrix theory is inherently quantum-mechanical and phase space plays an important role in the study of its compactifications. Secondly, supergravity, being a field theory, does not include the stringy winding modes. This is important because in non-geometric string backgrounds momentum modes and winding modes appear to be mixed. Such aspects can be addressed in the non-perturbative context of Matrix theory [59,60]. Finally, another advantage of Matrix theory over supergravity regards the quantization of flux. Indeed, while in string theory charges are quantized, in supergravity, being a classical field theory, the charges are continuous parameters [2]. On the contrary, in the context of Matrix theory we will determine appropriate quantization conditions.

\footnotetext{
${ }^{3}$ After the first version of this paper was posted, Ref. [46] appeared, where non-geometric fluxes in M theory are discussed as well within the framework of (M-theory extended) generalized geometry.
} 
The structure of the paper is as follows. In section 2 the matrix model of Banks, Fischler, Shenker and Susskind (BFSS) is briefly reviewed, along with its known compactifications on non-commutative tori and twisted tori. The issue of the description of non-geometric situations in Matrix theory is addressed in section 3. First, the algebraic building blocks responsible for the non-commutative/non-associative deformations are described by implementing the properties of twisted doubled tori in a matrix model framework. The way that each algebraic block can be obtained from another one indicates how T-duality operates on the corresponding solutions. In the process of relating such solutions we find a correspondence between position and momentum space which is reminiscent of a frame choice in generalized geometry. Furthermore, we obtain flux quantization conditions, a property that resolves the non-associativity of unitary operators. In section 4 certain aspects of the resulting gauge theories obtained from the compactifications of Matrix theory are discussed. Finally, we summarize our findings in section 5.

\section{The matrix model and its compactifications}

\subsection{The BFSS matrix model}

Let us begin by briefly describing the BFSS matrix model [1]. This model, also referred to as Matrix theory, was suggested as a non-perturbative definition of $\mathrm{M}$ theory. Its action, determining the dynamics of $N$ D0 branes in uncompactified spacetime, is given by the following functional:

$$
\mathcal{S}_{B F S S}=\frac{1}{2 g} \int d t\left[\operatorname{Tr}\left(\dot{\mathcal{X}}_{a} \dot{\mathcal{X}}_{a}-\frac{1}{2}\left[\mathcal{X}_{a}, \mathcal{X}_{b}\right]^{2}\right)+2 \psi^{T} \dot{\psi}-2 \psi^{T} \Gamma^{a}\left[\psi, \mathcal{X}_{a}\right]\right]
$$

where $\mathcal{X}_{a}(t), a=1, \ldots, 9$ are nine time-dependent $N \times N$ Hermitian matrices, $\psi$ are their fermionic superpartners and $\Gamma^{a}$ furnish a representation of $S O(9)$. In the limit of infinitedimensional matrices, namely $N \rightarrow \infty$, this matrix model is supposed to be equivalent to uncompactified $\mathrm{M}$ theory. In the following we shall be concerned mainly with the bosonic part of the above action. An important point is that this model may be thought of as supersymmetric quantum mechanics. As such it is inherently quantum-mechanical, which will play an important role in our discussions in the following sections.

The equations of motion resulting from the variation of the action (2.1) with respect to $\mathcal{X}_{a}$, setting $\psi=0$, are

$$
\ddot{\mathcal{X}}_{a}+\left[\mathcal{X}_{b},\left[\mathcal{X}^{b}, \mathcal{X}_{a}\right]\right]=0
$$

where indices are raised and lowered with $\delta_{a b}$ and therefore it does not make any difference whether they are upper or lower. For static configurations it is clear that the first term in (2.2) may be dropped.

\subsection{Compactification on tori}

A matrix compactification on a $d$-dimensional torus is defined by a restriction of the matrix action under certain periodicity conditions incorporating the cycles of the torus. Let us 
restrict to the 3-dimensional case, since most of our following discussions will relate to this number of dimensions. The generalization to any dimension is simple and straightforward.

For a $\mathrm{T}^{3}$ extending, say, in the directions $\mathcal{X}_{1}, \mathcal{X}_{2}, \mathcal{X}_{3}$, the compactification involves three invertible unitary matrices $U^{i}$ acting as translation operators and leading to the conditions

$$
\begin{aligned}
\mathcal{X}_{i}+R_{i} & =U^{i} \mathcal{X}_{i}\left(U^{i}\right)^{-1}, \quad i=1,2,3 \\
\mathcal{X}_{a} & =U^{i} \mathcal{X}_{a}\left(U^{i}\right)^{-1}, \quad a \neq i, \quad a=1, \ldots, 9
\end{aligned}
$$

where $R_{i}$ are constants. In the ensuing we set these constants to one since they may be easily reinserted at any point of the analysis.

It is well-known [16] that the conditions (2.3) are solved by

$$
\begin{aligned}
\mathcal{X}_{i} & =i \mathcal{D}_{i}, \quad \mathcal{X}_{m}=\mathcal{A}_{m}\left(\hat{U}^{i}\right), \quad m=4, \ldots, 9, \\
U^{i} & =e^{i \hat{x}^{i}}
\end{aligned}
$$

where the unitary operators satisfy $U^{i} U^{j}=\lambda^{i j} U^{j} U^{i}$ with complex constants $\lambda^{i j}=e^{-i \theta^{i j}}$, while the covariant derivatives have the form

$$
\hat{\mathcal{D}}_{i}=\hat{\partial}_{i}-i \mathcal{A}_{i}\left(\hat{U}^{j}\right)
$$

The special case of $\theta^{i j}=0$ or equivalently $\lambda^{i j}=1$ leads to commuting $U$ s, which correspond to the case of a standard 3-torus $\mathrm{T}^{3}$. However, in general the parameters $\theta^{i j}$ are not vanishing, in which case the $U$ s are not commuting operators. The latter case corresponds to a compactification on a non-commutative torus.

Let us note that $\mathcal{A}_{i}$ and $\mathcal{A}_{m}$ in (2.4) do not depend on $U \mathrm{~s}$, but rather on the set of operators $\hat{U}^{i}$ which commute with all $U^{i}$, i.e. $\left[\hat{U}^{i}, U^{j}\right]=0$. This has to be true in order for the conditions (2.3) to be satisfied. Of course, in the commutative case the $\hat{U}$ and the $U$ are identified. Moreover, a direct implication of the non-commutativity among the $U^{i}$ is that the $\hat{x}^{i}$ do not commute as well, but instead they satisfy the relation

$$
\left[\hat{x}^{i}, \hat{x}^{j}\right]=i \theta^{i j}
$$

Thus they may be interpreted as the coordinate operators of a non-commutative 3-torus $\mathrm{T}_{\theta}^{3}$, as long as they are periodic. In addition, in the present case the $\hat{U}_{\mathrm{s}}$ may be written as

$$
\hat{U}^{i}=e^{i \hat{x}^{i}+\theta^{i j} \hat{\partial}_{j}}
$$

and they satisfy the relations

$$
\hat{U}^{i} \hat{U}^{j}=e^{-i \hat{\theta}^{i j}} \hat{U}^{j} \hat{U}^{i}, \quad \hat{\theta}^{i j}=-\theta^{i j}
$$

Therefore, the compactification on a non-commutative torus leads to a gauge theory on a dual non-commutative torus with parameter $\hat{\theta}$. Indeed, substituting the solution back to the original matrix model action, one obtains a non-commutative supersymmetric YangMills theory on this dual torus [24]. 
Let us stress that the above considerations involve the full phase space of $\hat{x}^{i}$ and $\hat{p}_{i}=-i \hat{\partial}_{i}$ and that the phase space algebra reads as

$$
\begin{aligned}
& {\left[\hat{x}^{i}, \hat{x}^{j}\right]=i \theta^{i j},} \\
& {\left[\hat{x}^{i}, \hat{p}_{j}\right]=i \delta_{j}^{i},} \\
& {\left[\hat{p}_{i}, \hat{p}_{j}\right]=0 .}
\end{aligned}
$$

Note that the momenta are commutative and they satisfy the standard Heisenberg relation with the coordinates, while the latter exhibit constant non-commutativity.

The constant non-commutativity among the coordinate operators, which also controls the non-commutativity properties of the unitary operators $U^{i}$ and $\hat{U}^{i}$, has an interesting physical interpretation. It corresponds to turning on a background value for the 3 -form potential $C^{(3)}$ of 11-dimensional supergravity, which is the low-energy, field theory limit of M theory. Indeed, Connes, Douglas and Schwarz (CDS) suggested that that the deformation parameters $\theta^{i j}$ defining the non-commutative tori, correspond to moduli of the 11-dimensional supergravity, such that

$$
\left(\theta^{-1}\right)_{i j} \propto \int d x^{i} d x^{j} C_{i j-}^{(3)}
$$

where "-" denotes the light cone direction $x^{-}[16]$. In the language of the type IIA theory, which is obtained from 11-dimensional supergravity upon compactification on a circle, this relation may be written as

$$
\left(\theta^{-1}\right)_{i j} \propto \int d x^{i} d x^{j} B_{i j}
$$

where the NS-NS 2-form field $B$ of the type IIA supergravity is obtained by the 3-form $C^{(3)}$ in the compactification process. We will often denote such relations as

$$
\theta^{i j} \stackrel{C D S}{\longleftrightarrow} B_{i j}
$$

where the left hand side is related to the BFSS matrix model and the right hand side to type II backgrounds.

\subsection{Compactification on twisted tori}

Following the same reasoning as before, it is straightforward to define compactifications of Matrix theory on twisted tori. The simplest example of a twisted torus arises for $d=3$. In that case, a (twisted) compactification is achieved by imposing and solving an appropriately extended set of constraints, which involve again three unitary matrices $U^{1}, U^{2}, U^{3}$ and they $\operatorname{are}^{4}$

$$
\begin{aligned}
U^{i} \mathcal{X}_{i}\left(U^{i}\right)^{-1} & =\mathcal{X}_{i}+1, \quad i=1,2,3 \\
U^{1} \mathcal{X}_{3}\left(U^{1}\right)^{-1} & =\mathcal{X}_{3}-N \mathcal{X}_{2}, \\
U^{2} \mathcal{X}_{3}\left(U^{2}\right)^{-1} & =\mathcal{X}_{3}+N \mathcal{X}_{1}, \\
U^{i} \mathcal{X}_{a}\left(U^{i}\right)^{-1} & =\mathcal{X}_{a}, \quad a \neq i, \quad a=1, \ldots, 9, \quad(a, i) \neq\{(3,1),(3,2)\}
\end{aligned}
$$

\footnotetext{
${ }^{4}$ Note that these constraints are slightly different than the ones presented in [18]. This is nothing but an equivalent description of the 3-dimensional twisted torus which will render our present discussion more practical.
} 
The latter constraints generalize the ones for the square torus appearing in (2.3), thus incorporating the twist of the 3-dimensional nilmanifold $\tilde{\mathrm{T}}^{3}[17,18]$. A general solution of the above constraints is given again by Eq. (2.4), with the following new features:

- The operators $U^{i}$ satisfy the relation

$$
U^{i} U^{j}=e^{-i \theta^{i j}-i N f_{k}^{i j} \hat{x}^{k}} U^{j} U^{i}
$$

where $f_{k}^{i j}=f_{k}^{i j}$ are antisymmetric only in the upper two indices and the only non-vanishing components are $f_{3}^{12}=-f_{3}^{21}=1$. In fact they correspond to the structure constants of the unique nilpotent Lie algebra in three dimensions, which plays an important role in the construction of the twisted torus [18]. Moreover, these parameters are also known as geometric fluxes in the language of Scherk-Schwarz compactifications [30]. The relation (2.14) defines a non-commutative twisted torus.

- The components $\mathcal{A}_{i}$ must be corrected to $\hat{\mathcal{A}}_{i}=\mathcal{A}_{i}+i N f_{i}{ }^{j k} \mathcal{A}_{j} \hat{\partial}_{k}$, which in the present case means that only $\mathcal{A}_{3}$ has to be corrected accordingly.

- For the above solution we can find the set of $\hat{U}_{\mathrm{s}}$ which provide the dependence of $\mathcal{A}_{i}$ and $\mathcal{A}_{m}$ and thus give the connection on a trivial gauge bundle. They have the form $\hat{U}^{i}=e^{i \hat{y}^{i}}$, where

$$
\hat{y}^{i}=\hat{x}^{i}-i \theta^{i j} \hat{\partial}_{j}-i N f_{k}^{i j} \hat{x}^{k} \hat{\partial}_{j}
$$

As before, these operators commute with the $U^{i}$ by construction ${ }^{5}$. Moreover, they satisfy the commutation relations of a dual non-commutative twisted torus, namely

$$
\hat{U}^{i} \hat{U}^{j}=e^{i \theta^{i j}+i N f_{k}^{i j} \hat{x}^{k}} \hat{U}^{j} \hat{U}^{i}
$$

As before, substituting the solution back to the original matrix model action we obtain a gauge theory on this dual non-commutative twisted torus. We will return to this point later in this paper.

\footnotetext{
${ }^{5} \mathrm{An}$ important note on notation is in order. The operators $U^{i}$ and $\hat{U}^{i}$ act on a Hilbert space of states $\mathcal{H}$. Let an arbitrary element of $\mathcal{H}$ be $f\left(\hat{x}^{i}\right)$. Then the corresponding actions (for $N=1$ ) are

$$
\left(U^{i} f\right)\left(\hat{x}^{j}\right)=e^{i \hat{x}^{i}} f\left(\hat{x}^{j}\right)
$$

and

$$
\left(\hat{U}^{i} f\right)\left(\hat{x}^{j}\right)=e^{i \hat{x}^{i}} f\left(\hat{x}^{j}+\theta^{i j}+f_{k}^{i j} \hat{x}^{k}\right) .
$$

Therefore a simple computation gives

$$
\left(U^{i} \hat{U}^{j} f\right)\left(\hat{x}^{l}\right)=\left(U^{i} e^{i \hat{x}^{j}} f\right)\left(\hat{x}^{l}+\theta^{j l}+f_{k}^{j l} \hat{x}^{k}\right)=e^{i \hat{x}^{i}} e^{i \hat{x}^{j}} f\left(\hat{x}^{l}+\theta^{j l}+f_{k}^{j l} \hat{x}^{k}\right),
$$

while

$$
\left(\hat{U}^{j} U^{i} f\right)\left(\hat{x}^{l}\right)=\left(\hat{U}^{j} e^{i \hat{x}^{i}} f\right)\left(\hat{x}^{l}\right)=e^{i \hat{x}^{j}} e^{i \hat{x}^{i}+i \theta^{j i}+i f^{j i}{ }_{k} \hat{x}^{k}} f\left(\hat{x}^{l}+\theta^{j l}+f_{k}^{j l} \hat{x}^{k}\right) .
$$

It is now easy to see that the Baker-Campbell-Hausdorff formula leads to the desired commutation between $U^{i}$ and $\hat{U}^{i}$. Note that had we just computed the commutator between $\hat{x}^{i}$ and $\hat{y}^{i}$ we would have obtained an incorrect result, having missed the specific way that these operators act on states of the Hilbert space. This subtlety should be kept in mind for all similar computations involving $U$-operators in this paper.
} 
- Finally, the algebra of the phase space is determined to be

$$
\begin{aligned}
{\left[\hat{x}^{i}, \hat{x}^{j}\right] } & =i \theta^{i j}+i N f_{k}^{i j} \hat{x}^{k} \\
{\left[\hat{p}_{i}, \hat{p}_{j}\right] } & =0 \\
{\left[\hat{p}_{i}, \hat{x}^{j}\right] } & =-i \delta_{i}^{j}-i N f_{i}{ }^{j k} \hat{p}_{k} .
\end{aligned}
$$

It is directly observed that the momenta remain commutative, however the (previously constant) non-commutativity of the coordinates acquires a non-constant part. This is exactly a (nilpotent) Lie algebra type non-commutativity.

Needless to mention that the formulae of the present paragraph are general, in the sense that they retain the same form for the compactification on any higher-dimensional nilmanifold. For example, a six-dimensional case was explicitly presented in [18], where all the necessary data for a large class of higher-dimensional nilmanifolds may be found as well (see also Ref. [61]).

A final comment on the case of twisted tori regards the Connes-Douglas-Schwarz correspondence. In the present case this translates into the statement that the non-constant non-commutativity of the coordinate operators, controlling also the non-commutative properties of the operators $U^{i}$ and $\hat{U}^{i}$, corresponds to turning on a geometric flux in 11dimensional/type IIA supergravity [31-33]. Schematically this means that

$$
\theta^{i j}(\hat{x}) \stackrel{C D S}{\longleftrightarrow} f_{i j}{ }^{k}
$$

This relation is supported by the resulting theory, as we will discuss in section 4 .

\section{Non-geometric compactifications of Matrix theory}

In the previous section we remembered some of the established compactifications of Matrix theory on non-commutative tori and twisted tori. We already mentioned that these correspond to compactifications in the presence of a background B-field or geometric fluxes respectively. The natural question which immediately arises regards the implementation of other types of fluxes in this scheme. The most standard one is of course the NS-NS flux, which from the point of view of twisted tori corresponds to a T-dual background with respect to the geometric flux one. However, it is by now well-known that there exist backgrounds with non-geometric fluxes formally obtained by further T-dualities of the geometric flux background. In this section we are going to describe Matrix theory flux compactifications with NS-NS $H$ flux, as well as non-geometric $Q$ and $R$ fluxes.

Let us begin with the following observation. In the compactifications of section 2 it is evident that apart from non-commutative coordinate operators it is necessary to consider momentum operators as well. This leads naturally to a phase space description, reminiscent of the quantum-mechanical structure of Matrix theory. However, in section 2 we did not introduce the unitary operators

$$
\tilde{U}_{i}=e^{i \hat{p}_{i}}
$$


which are the counterparts of the operators $U^{i}$ in the phase space. Of course, the reason that they were not introduced previously is that in the case of the twisted torus they commute among themselves, as well as with the $\mathcal{X}_{i}$ and therefore they did not play any crucial role up to now. In the present section we introduce these operators in order to exploit the full potential of the phase space.

According to the above, there is now a second set of unitary operators acting on $\mathcal{X}_{i}$. This reminds us of the doubled formalism, which was used in order to construct the so-called twisted doubled tori and provide an adequate description of non-geometric situations in terms of the geometry of an enlarged space. This realization motivates us to introduce also a set of "dual" Hermitian matrices $\tilde{\mathcal{X}}^{i}$ and impose on the matrices $\mathcal{X}_{i}$ and $\tilde{\mathcal{X}}^{i}$ the conditions which describe a compactification on a twisted doubled torus. Subsequently, we will utilize this formulation in order to describe the non-geometric compactifications and we will discuss the structure of the resulting theories.

\subsection{Algebraic building blocks for fluxes}

Here we are going to define and solve the conditions corresponding to a flux compactification of Matrix theory with $f, H, Q$ and $R$ flux. Technically the first one was already done in section 2 , but we shall reformulate it in terms of twisted doubled tori and in a way it will serve as a calibration of our formulation.

In all the following cases our solutions will have the form

$$
\begin{aligned}
\mathcal{X}_{i} & =i \hat{\partial}_{i}+\hat{\mathcal{A}}_{i}, \\
\tilde{\mathcal{X}}^{i} & =(-1)^{c_{i}} \hat{x}^{i}+\hat{\tilde{\mathcal{A}}}^{i},
\end{aligned}
$$

where the hat over the $\mathcal{A}$ and $\tilde{\mathcal{A}}$ denotes that these components may have to be appropriately corrected. In the second line we introduced a grading $(-1)^{c_{i}}$, which has to be included in order to guarantee that the Heisenberg relation is not spoiled. Its values will be given case by case in the following analysis. Moreover, the unitary operators will always have the form

$$
\begin{aligned}
& U^{i}=e^{i \hat{x}^{i}}, \\
& \tilde{U}_{i}=e^{(-1)^{c_{i}} \hat{\partial}_{i}},
\end{aligned}
$$

with the same grading as above. Finally, in the present section we set the constant part of the non-commutativity to zero, $\theta^{i j}=0$. These parameters may be reintroduced if necessary.

The $f$-block. This is the geometric flux situation, corresponding to a specific twisted doubled torus as described in [36,37]. Here we follow the conventions of [37]. The conditions that define the specific compactification in the matrix model framework are the following:

$$
\begin{aligned}
U^{i} \mathcal{X}_{i}\left(U^{i}\right)^{-1} & =\mathcal{X}_{i}+1 \\
U^{1} \mathcal{X}_{3}\left(U^{1}\right)^{-1} & =\mathcal{X}_{3}-\mathcal{X}_{2} \\
U^{2} \mathcal{X}_{3}\left(U^{2}\right)^{-1} & =\mathcal{X}_{3}+\mathcal{X}_{1}
\end{aligned}
$$


and

$$
\begin{aligned}
\tilde{U}_{i} \tilde{\mathcal{X}}^{i}\left(\tilde{U}_{i}\right)^{-1} & =\tilde{\mathcal{X}}^{i}+1, \\
\tilde{U}_{3} \tilde{\mathcal{X}}^{1}\left(\tilde{U}_{3}\right)^{-1} & =\tilde{\mathcal{X}}^{1}+\mathcal{X}_{2}, \\
\tilde{U}_{3} \tilde{\mathcal{X}}^{2}\left(\tilde{U}_{3}\right)^{-1} & =\tilde{\mathcal{X}}^{2}-\mathcal{X}_{1}, \\
U^{1} \tilde{\mathcal{X}}^{2}\left(U^{1}\right)^{-1} & =\tilde{\mathcal{X}}^{2}+\tilde{\mathcal{X}}^{3} \\
U^{2} \tilde{\mathcal{X}}^{1}\left(U^{2}\right)^{-1} & =\tilde{\mathcal{X}}^{1}-\tilde{\mathcal{X}}^{3}
\end{aligned}
$$

while all the combinations that do not appear in the above equations are supposed to have the trivial form $U \mathcal{X} U^{-1}=\mathcal{X}$ and similarly for the tilded ones. We directly observe that the first conditions, Eq. (3.3), are the same that we encountered in section 2 for the twisted torus.

When the unitary operators have the form (3.2), then the connections appearing in Eq. (3.1) solve all the compactification conditions under the following requirements:

- The grading exponent $c_{i}$ has the value 1 for $i=3$ and it is zero for $i \neq 3$.

- The algebra of $\left\{\hat{x}^{i}, \hat{\partial}_{i}\right\}$ has the form

$$
\begin{aligned}
& {\left[\hat{x}^{i}, \hat{x}^{j}\right]=i f^{i j}{ }_{k} \hat{x}^{k},} \\
& {\left[\hat{\partial}_{i}, \hat{\partial}_{j}\right]=0,} \\
& {\left[\hat{\partial}_{i}, \hat{x}^{j}\right]=\delta_{i}^{j}-i f_{i}{ }^{j k} \hat{\partial}_{k},}
\end{aligned}
$$

where only $f_{3}^{12}=-f_{3}^{21}=1$ are non-vanishing. This is identical to the algebra we determined for the single twisted torus in Eq. (2.17) for $\theta^{i j}=0$ and $N=1$, as expected.

- The gauge fields $\hat{\mathcal{A}}_{i}$ and $\hat{\tilde{\mathcal{A}}}^{i}$ are given by

$$
\begin{aligned}
\hat{\mathcal{A}}_{i} & =\mathcal{A}_{i}+i f_{i}{ }^{j k} \mathcal{A}_{j} \hat{\partial}_{k}, \\
\tilde{\tilde{\mathcal{A}}}^{i} & =\tilde{\mathcal{A}}^{i}-f_{j}{ }^{i k} \mathcal{A}_{k} \hat{x}^{j} .
\end{aligned}
$$

Note that $\mathcal{A}_{i}$ are functions of a set of operators $\hat{U}^{i}$ with the property $\left[\hat{U}^{i}, U^{i}\right]=0$.

The above requirements fix the $U$ - and $\tilde{U}$-algebra and moreover allow us to determine the operators $\hat{U}^{i}$ and their algebra. Indeed we find

$$
\begin{aligned}
U^{i} U^{j} & =e^{-i f_{k}^{i j} \hat{x}^{k}} U^{j} U^{i}=f_{k}^{i j}\left(U^{k}\right)^{-1} U^{j} U^{i}, \\
\tilde{U}_{i} \tilde{U}_{j} & =\tilde{U}_{j} \tilde{U}_{i}, \\
U^{i} \tilde{U}_{j} & =e^{-(-1)^{c_{j}} f_{j}{ }^{i k} \hat{\partial}_{k}} \tilde{U}_{j} U^{i} .
\end{aligned}
$$

Moreover, the $\hat{U}^{i}$ and their algebra are identical to the ones appearing in Eqs. (2.15) and (2.16) respectively, with $\theta^{i j}=0$. 
Let us now make an important observation concerning the phase space algebra (3.5). It is simple to check that it is in fact non-associative. One way to see this is by calculating the double commutators

$$
-1=\left[\left[\hat{p}_{3}, \hat{x}^{1}\right], \hat{x}^{2}\right] \neq\left[\hat{p}_{3},\left[\hat{x}^{1}, \hat{x}^{2}\right]\right]=1 .
$$

Alternatively, the Jacobiator with one $\hat{p}$ and two $\hat{x}$ entries does not vanish:

$$
\left[\hat{p}_{i}, \hat{x}^{j}, \hat{x}^{k}\right] \equiv\left[\left[\hat{p}_{i}, \hat{x}^{j}\right], \hat{x}^{k}\right]+\text { c.p. }=-3 f_{i}^{j k} \text {. }
$$

In our framework this is a quantum-mechanical property, since it is a consequence of the Heisenberg relation between the coordinate and momentum operators. Upon introducing the quantum of action $\hbar$, it will appear multiplicatively on the right hand side of (3.9). Thus, as $\hbar \rightarrow 0$ this non-associativity will go away. Moreover, let us stress that the dynamical degrees of freedom, $\mathcal{X}$ and $\mathcal{A}$, do not exhibit this type of non-associativity. Further discussion on this point is left for section 3.4.

As we already mentioned the extended structure introduced here does not add much to the results of the single twisted torus. Indeed, projecting to the relevant sector of $\mathcal{X}_{i}$ we obtain the geometric flux compactification of Matrix theory. However, the present formulation turns out to be very useful in order to account for other types of fluxes, as we will immediately do.

The $H$-block. In this paragraph we are going to describe a doubled compactification which will capture the case of a torus with $H$ flux. Such a compactification was described in $[36,37]$ in the supergravity picture. Along the lines of the $f$-block, this twisted doubled torus compactification is described in the matrix model with the following non-trivial conditions on $\mathcal{X}_{i}$ and $\tilde{\mathcal{X}}^{i}$ :

$$
\begin{aligned}
& U^{i} \mathcal{X}_{i}\left(U^{i}\right)^{-1}=\mathcal{X}_{i}+1 \\
& \tilde{U}_{i} \tilde{\mathcal{X}}^{i}\left(\tilde{U}_{i}\right)^{-1}=\tilde{\mathcal{X}}^{i}+1
\end{aligned}
$$

and

$$
U^{i} \tilde{\mathcal{X}}^{j}\left(U^{i}\right)^{-1}=\tilde{\mathcal{X}}^{j}+H^{i j k} \mathcal{X}_{k}
$$

where $H^{i j k}$ is fully antisymmetric and $H^{123}=1$.

When the unitary operators have the form (3.2), we obtain a full solution as in Eq. (3.1) under the following requirements:

- Concerning the grading exponent: $c_{i}=0$ for every $i=1,2,3$.

- The algebra of $\left\{\hat{x}^{i}, \hat{\partial}_{i}\right\}$ has the form

$$
\begin{aligned}
& {\left[\hat{x}^{i}, \hat{x}^{j}\right]=H^{i j k} \hat{\partial}_{k},} \\
& {\left[\hat{\partial}_{i}, \hat{\partial}_{j}\right]=0} \\
& {\left[\hat{\partial}_{i}, \hat{x}^{j}\right]=\delta_{i}^{j} .}
\end{aligned}
$$


- The gauge fields $\hat{\mathcal{A}}_{i}$ and $\hat{\tilde{\mathcal{A}}}^{i}$ are given by

$$
\begin{aligned}
\hat{\mathcal{A}}_{i} & =\mathcal{A}_{i}, \\
\tilde{\tilde{\mathcal{A}}}^{i} & =\tilde{\mathcal{A}}^{i}+i H^{i j k} \mathcal{A}_{j} \hat{\partial}_{k},
\end{aligned}
$$

i.e. $\mathcal{A}_{i}$ is not modified. Note that, as always, the $\mathcal{A}_{i}$ are functions of a set of operators $\hat{U}^{i}$ with the property $\left[\hat{U}^{i}, U^{i}\right]=0$.

According to the above, the algebras of $U^{i}$ and $\tilde{U}^{i}$ are now fixed to be

$$
\begin{aligned}
U^{i} U^{j} & =e^{-H^{i j k} \hat{\partial}_{k}} U^{j} U^{i}=H^{i j k}\left(\tilde{U}^{k}\right)^{-1} U^{j} U^{i}, \\
\tilde{U}_{i} \tilde{U}_{j} & =\tilde{U}_{j} \tilde{U}_{i}, \\
U^{i} \tilde{U}_{j} & =\tilde{U}_{j} U^{i} .
\end{aligned}
$$

Moreover the operators $\hat{U}^{i}$, which centralize the $U^{i}$ ones, are

$$
\hat{U}^{i}=e^{i x^{i}+i H^{i j k} \hat{\partial}_{j} \hat{\partial}_{k}},
$$

and they satisfy the dual relations

$$
\hat{U}^{i} \hat{U}^{j}=e^{H^{i j k} \hat{\partial}_{k}} \hat{U}^{j} \hat{U}^{i}=\left(H^{i j k} \tilde{U}^{k}\right) \hat{U}^{j} \hat{U}^{i} .
$$

This non-commutativity should be related to a non-vanishing background B-field. Indeed, the commutativity of the gauge algebra is now obstructed by the parameters

$$
\theta^{i j}=H^{i j k} \hat{p}_{k} .
$$

Then, the relation $\theta^{-1} \sim B$ dictates that the corresponding IIA supergravity is compactified on a torus with non-constant $\mathrm{B}$ field:

$$
B=x^{1} d x^{2} \wedge d x^{3}+x^{2} d x^{3} \wedge d x^{1}+x^{3} d x^{1} \wedge d x^{2},
$$

where $x^{i}$ are the toroidal coordinates. This is in accord with the results of Ref. [37].

A similar observation to the previous case is that the phase space algebra (3.12) is also non-associative. Indeed, the Jacobiator with three $\hat{x}$ entries does not vanish:

$$
\left[\hat{x}^{i}, \hat{x}^{j}, \hat{x}^{k}\right]=3 H^{i j k} .
$$

It is very welcome that the Jacobiator is obstructed exactly by the object $H$, which is associated to the NS-NS flux. Note again that the dynamical variables $\mathcal{X}_{i}$ do not feel this non-associativity (see also section 3.4).

The $Q$-block. A further possibility arises when we consider the twisted doubled torus of Ref. [37] which is related to a non-geometric $Q$ flux. This translates in the matrix model into the following non-trivial compactification conditions:

$$
\begin{aligned}
U^{i} \mathcal{X}_{i}\left(U^{i}\right)^{-1} & =\mathcal{X}_{i}+1, \\
U^{1} \mathcal{X}_{2}\left(U^{1}\right)^{-1} & =\mathcal{X}_{2}+\tilde{\mathcal{X}}^{3}, \\
U^{1} \mathcal{X}_{3}\left(U^{1}\right)^{-1} & =\mathcal{X}_{3}-\tilde{\mathcal{X}}^{2},
\end{aligned}
$$


and

$$
\begin{aligned}
\tilde{U}_{i} \tilde{\mathcal{X}}^{i}\left(\tilde{U}_{i}\right)^{-1} & =\tilde{\mathcal{X}}^{i}+1, \\
\tilde{U}_{2} \mathcal{X}_{3}\left(\tilde{U}_{2}\right)^{-1} & =\mathcal{X}_{3}+\mathcal{X}_{1}, \\
\tilde{U}_{3} \mathcal{X}_{2}\left(\tilde{U}_{3}\right)^{-1} & =\mathcal{X}_{2}-\mathcal{X}_{1}, \\
\tilde{U}_{2} \tilde{\mathcal{X}}^{1}\left(\tilde{U}_{2}\right)^{-1} & =\tilde{\mathcal{X}}^{1}-\tilde{\mathcal{X}}^{3}, \\
\tilde{U}_{3} \tilde{\mathcal{X}}^{1}\left(\tilde{U}_{3}\right)^{-1} & =\tilde{\mathcal{X}}^{1}+\tilde{\mathcal{X}}^{2},
\end{aligned}
$$

With the unitary operators of Eq. (3.2) and the solution of Eq. (3.1), the corresponding requirements are:

- The grading exponent has the values $c_{2}=c_{3}=1$ and $c_{1}=0$.

- The algebra of $\left\{\hat{x}^{i}, \hat{\partial}_{i}\right\}$ has the form

$$
\begin{aligned}
{\left[\hat{x}^{i}, \hat{x}^{j}\right] } & =0, \\
{\left[\hat{\partial}_{i}, \hat{\partial}_{j}\right] } & =Q_{i j}{ }^{k} \hat{\partial}_{k}, \\
{\left[\hat{\partial}_{i}, \hat{x}^{j}\right] } & =\delta_{i}^{j}-Q_{i k}{ }^{j} \hat{x}^{k} .
\end{aligned}
$$

where $Q_{i j}{ }^{k}$ is antisymmetric in its lower two indices and the only non-vanishing index structure is $Q_{23}{ }^{1}=1$. We directly observe the novel feature that the momenta are now non-commutative, while presently the coordinates commute among themselves.

- The gauge fields $\hat{\mathcal{A}}_{i}$ and $\hat{\tilde{\mathcal{A}}}^{i}$ are given by

$$
\begin{aligned}
\hat{\mathcal{A}}_{i} & =\mathcal{A}_{i}+i Q_{i j}{ }^{k} \tilde{\mathcal{A}}_{j} \hat{\partial}^{k} \\
\hat{\tilde{\mathcal{A}}}^{i} & =\tilde{\mathcal{A}}^{i}+Q_{j k}{ }^{i} \tilde{\mathcal{A}}^{k} \hat{x}^{j}
\end{aligned}
$$

The $U$ - and $\tilde{U}$-algebra now becomes

$$
\begin{aligned}
U^{i} U^{j} & =U^{j} U^{i}, \\
\tilde{U}_{i} \tilde{U}_{j} & =e^{Q_{i j}{ }^{k} \hat{\partial}_{k}} \tilde{U}_{j} \tilde{U}_{i}=\left(Q_{i j}{ }^{k} \tilde{U}_{k}\right) \tilde{U}_{j} \tilde{U}_{i}, \\
U^{i} \tilde{U}_{j} & =e^{-i Q_{j k}{ }^{i} \hat{x}^{k}} \tilde{U}_{j} U^{i},
\end{aligned}
$$

where we observe that the operators $U^{i}$ now commute among themselves. This obviates the need to introduce the corresponding hatted operators $\hat{U}^{i}$. On the other hand, unlike the previous cases, now the $\tilde{U}_{i}$ operators do not commute among themselves. Therefore, for later use, it is instructive to introduce the hatted operators which commute with them, i.e. $\left[\hat{\tilde{U}}_{i}, \tilde{U}_{j}\right]=0$. These operators read as

$$
\hat{\tilde{U}}_{i}=e^{(-1)^{c_{i}} \hat{\partial}_{i}-Q_{i j}{ }^{k} \hat{x}^{j} \hat{\partial}_{k}}
$$

and they satisfy an algebra dual to the one of $\tilde{U}_{i}$, namely

$$
\hat{\tilde{U}}_{i} \hat{\tilde{U}}_{j}=e^{-Q_{i j}{ }^{k}} \hat{\partial}_{k} \hat{\tilde{U}}_{j} \hat{\tilde{U}}_{i}=Q_{i j}^{k}\left(\hat{\tilde{U}}_{k}\right)^{-1} \hat{\tilde{U}}_{j} \hat{\tilde{U}}_{i}
$$


As before, there is a non-associativity related to the algebra of the phase space. The relevant Jacobiator in the present case involves one $\hat{x}$ and two $\hat{p}$ entries and it reads as

$$
\left[\hat{x}^{i}, \hat{p}_{j}, \hat{p}_{k}\right]=3 Q^{i}{ }_{j k}
$$

We observe that the Jacobiator is now obstructed exactly by $Q$. A novel feature is that here the dynamical matrices $\mathcal{X}_{i}$ do not commute among themselves. This will be discussed in the subsection 3.3.

The $R$-block. The last algebraic block in this series should be related to the $R$ flux, which is captured by the appropriate twisted doubled torus. The compactification conditions implementing the geometry of this torus now read as

$$
\begin{aligned}
& U^{i} \mathcal{X}_{i}\left(U^{i}\right)^{-1}=\mathcal{X}_{i}+1 \\
& \tilde{U}_{i} \tilde{\mathcal{X}}^{i}\left(\tilde{U}_{i}\right)^{-1}=\tilde{\mathcal{X}}^{i}+1
\end{aligned}
$$

and

$$
\tilde{U}_{i} \mathcal{X}_{j}\left(\tilde{U}_{i}\right)^{-1}=\mathcal{X}_{j}+R_{i j k} \tilde{\mathcal{X}}_{k}
$$

The usual requirements which fix the solution now are:

- Grading exponent: $c_{i}=1$ for all $i=1,2,3$.

- The algebra of $\left\{\hat{x}^{i}, \hat{\partial}_{i}\right\}$ has the form

$$
\begin{aligned}
& {\left[\hat{x}^{i}, \hat{x}^{j}\right]=0} \\
& {\left[\hat{\partial}_{i}, \hat{\partial}_{j}\right]=-i R_{i j k} \hat{x}^{k}} \\
& {\left[\hat{\partial}_{i}, \hat{x}^{j}\right]=\delta_{i}^{j} .}
\end{aligned}
$$

- The gauge fields $\hat{\mathcal{A}}_{i}$ and $\tilde{\hat{\mathcal{A}}}^{i}$ are given by

$$
\begin{aligned}
\hat{\mathcal{A}}_{i} & =\mathcal{A}_{i}-R_{i j k} \tilde{\mathcal{A}}^{j} \hat{x}^{k}, \\
\tilde{\hat{\mathcal{A}}}^{i} & =\tilde{\mathcal{A}}^{i} .
\end{aligned}
$$

The algebra of $U^{i}$ and $\tilde{U}_{i}$ operators is now found to be

$$
\begin{aligned}
U^{i} U^{j} & =U^{j} U^{i}, \\
\tilde{U}_{i} \tilde{U}_{j} & =e^{-i R_{i j k} \hat{x}^{k}} \tilde{U}_{j} \tilde{U}_{i}, \\
U^{i} \tilde{U}_{j} & =\tilde{U}_{j} U^{i} .
\end{aligned}
$$

Similarly to the $Q$-block, the $U^{i}$ commute among themselves, unlike the $\tilde{U}^{i}$. Therefore we introduce the operators centralizing the latter, i.e.

$$
\hat{\tilde{U}}_{i}=e^{-\hat{\partial}_{i}-i R_{i j k} \hat{x}^{j} \hat{x}^{k}},
$$


which satisfy the dual algebra

$$
\hat{\tilde{U}}_{i} \hat{\tilde{U}}_{j}=e^{i R_{i j k} \hat{x}^{k}} \hat{\tilde{U}}_{j} \hat{\tilde{U}}_{i}
$$

Finally, the non-associativity related to the phase space algebra may be traced in the Jacobiator involving three $\hat{p}$ entries. Indeed we compute

$$
\left[\hat{p}_{i}, \hat{p}_{j}, \hat{p}_{k}\right]=3 R_{i j k},
$$

thus obtaining an obstruction by $R$. Moreover, note that in the present case the dynamical matrices $\mathcal{X}_{i}$ not only do not commute among themselves but they do not associate as well. This is part of the discussion which follows in subsection 3.3.

\subsection{Moving from block to block - T-duality}

The four algebras which we obtained for each flux-block appear in Eqs. (3.5),(3.12),(3.21) and (3.29). Let us now explore how starting with one of them we can obtain all the rest with appropriate transformations. Our starting point is the $H$-block which moreover is the leftmost entry in the T-duality chain (1.1). It is directly observed that the algebra (3.5) of the $f$-block is obtained by the $H$-block algebra (3.12) under the canonical transformation

$$
\begin{aligned}
& \hat{x}^{3} \rightarrow-\hat{p}_{3}, \\
& \hat{p}_{3} \rightarrow \hat{x}^{3} .
\end{aligned}
$$

Equivalently, we may rewrite this transformation as

$$
\left(\begin{array}{c}
\hat{x}^{3} \\
\hat{p}_{3}
\end{array}\right) \rightarrow\left(\begin{array}{cc}
0 & -1 \\
1 & 0
\end{array}\right)\left(\begin{array}{c}
\hat{x}^{3} \\
\hat{p}_{3}
\end{array}\right)=-i \sigma_{2}\left(\begin{array}{c}
\hat{x}^{3} \\
\hat{p}_{3}
\end{array}\right) .
$$

Similarly, we can move from the algebra of the $f$-block to the algebra (3.21) of the $Q$-block by means of the canonical transformation

$$
\begin{aligned}
& \hat{x}^{2} \rightarrow-\hat{p}_{2}, \\
& \hat{p}_{2} \rightarrow \hat{x}^{2},
\end{aligned}
$$

while the transformation from the $Q$-block algebra to the $R$-block one is similarly given by

$$
\begin{aligned}
& \hat{x}^{1} \rightarrow-\hat{p}_{1}, \\
& \hat{p}_{1} \rightarrow \hat{x}^{1} .
\end{aligned}
$$

Defining the six-dimensional column vector

$$
\hat{q}^{i}:=\left(\begin{array}{c}
\hat{x}^{i} \\
\hat{p}_{i}
\end{array}\right),
$$

the above transformations are realized by the following six-dimensional matrices

$$
M_{H \rightarrow f}=\left(\begin{array}{cccccc}
1 & 0 & 0 & 0 & 0 & 0 \\
0 & 1 & 0 & 0 & 0 & 0 \\
0 & 0 & 0 & 0 & 0 & -1 \\
0 & 0 & 0 & 1 & 0 & 0 \\
0 & 0 & 0 & 0 & 1 & 0 \\
0 & 0 & 1 & 0 & 0 & 0
\end{array}\right), M_{f \rightarrow Q}=\left(\begin{array}{cccccc}
1 & 0 & 0 & 0 & 0 & 0 \\
0 & 0 & 0 & -1 & 0 & 0 \\
0 & 0 & 1 & 0 & 0 & 0 \\
0 & 0 & 0 & 1 & 0 & 0 \\
0 & 1 & 0 & 0 & 0 & 0 \\
0 & 0 & 0 & 0 & 0 & 1
\end{array}\right),
$$


and

$$
M_{Q \rightarrow R}=\left(\begin{array}{cccccc}
0 & 0 & 0 & -1 & 0 & 0 \\
0 & 1 & 0 & 0 & 0 & 0 \\
0 & 0 & 1 & 0 & 0 & 0 \\
1 & 0 & 0 & 0 & 0 & 0 \\
0 & 0 & 0 & 0 & 1 & 0 \\
0 & 0 & 0 & 0 & 0 & 1
\end{array}\right)
$$

respectively. Additionally, one may define the matrix which directly connects the starting $H$-block to the $R$-block. This is just the multiplication of the above three matrices and it is given as

$$
M_{H \rightarrow R}=\left(\begin{array}{cccccc}
0 & 0 & 0 & -1 & 0 & 0 \\
0 & 0 & 0 & 0 & -1 & 0 \\
0 & 0 & 0 & 0 & 0 & -1 \\
1 & 0 & 0 & 0 & 0 & 0 \\
0 & 1 & 0 & 0 & 0 & 0 \\
0 & 0 & 1 & 0 & 0 & 0
\end{array}\right)
$$

The above phase space transformations connect the algebraic blocks in the way we discussed. On the other hand we know that the solutions corresponding to each block should be related among themselves by T-dualities, in the spirit of the chain (1.1). However, it is evident that the above matrices are not elements of the T-duality group. Thus, these canonical transformations are not directly associated to T-dualities. At this point the grading operator that we introduced in (3.1) comes into play. Let us consider the unitary operators $U^{i}$ and $\tilde{U}_{i}$ on equal footing by the column

$$
\left(\begin{array}{c}
U^{i} \\
\tilde{U}_{i}
\end{array}\right)=\left(\begin{array}{c}
e^{i \hat{x}^{i}} \\
e^{(-1)^{c_{i} i \hat{p}_{i}}}
\end{array}\right):=e^{(-1)^{\hat{c}_{i} i \hat{q}^{i}}},
$$

where we introduced the grading operator $(-1)^{\hat{c}_{i}}$, representing the action of $(-1)^{c_{i}}$ on all the unitary operators. If we represent this grading operator by a six-dimensional matrix acting on the column vector $\hat{q}^{i}$, then for the $H$-block it is just

$$
(-1)_{H}^{\hat{c}_{i}}=\mathbb{1}_{6},
$$

where $\mathbb{1}_{6}$ denotes the unit matrix in six dimensions. For the $f$-block this operator has the diagonal form

$$
(-1)_{f}^{\hat{c}_{i}}=\operatorname{diag}(1,1,1,1,1,-1),
$$

and similarly for the other two blocks,

$$
\begin{aligned}
& (-1)_{Q}^{\hat{c}_{i}}=\operatorname{diag}(1,1,1,1,-1,-1) \\
& (-1)_{R}^{\hat{c}_{i}}=\operatorname{diag}(1,1,1,-1,-1,-1) .
\end{aligned}
$$

Therefore we now have at hand two operations, namely the canonical transformations given by the matrices $M$, which connect just the algebraic building blocks, and the gradings $(-1)^{\hat{c}_{i}}$, which preserve the Heisenberg relation. When we move from one block to the other it is the combined action of these two operations that connects one solution to another. Let 
us consider for example the case of moving from the $H$ - to the $f$-block. The corresponding operation is

$$
M_{H \rightarrow f} \cdot(-1)_{f}^{\hat{c}_{i}}=\left(\begin{array}{cccccc}
1 & 0 & 0 & 0 & 0 & 0 \\
0 & 1 & 0 & 0 & 0 & 0 \\
0 & 0 & 0 & 0 & 0 & 1 \\
0 & 0 & 0 & 1 & 0 & 0 \\
0 & 0 & 0 & 0 & 1 & 0 \\
0 & 0 & 1 & 0 & 0 & 0
\end{array}\right):=T_{3} .
$$

The latter, $T_{3}$, is an element of the compact subgroup of the T-duality group and generates (at least formally) a T-duality along the $x^{3}$ direction of the torus, thus leading to the corresponding twisted torus without $H$ flux. This way one can explain the T-duality chain (1.1) in the present framework.

The above results can be represented as in the following diagram:

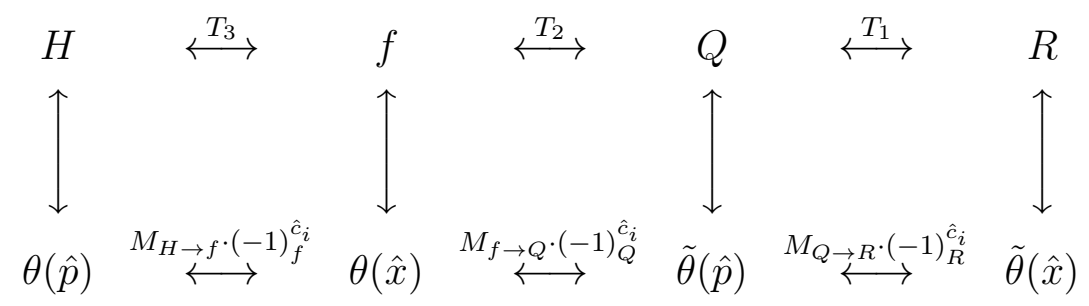

The vertical arrows denote the CDS correspondence, i.e. they connect a compactification of the matrix model to a compactification of low-energy supergravity. The upper horizontal arrows denote T-duality among supergravity vacua, while the lower horizontal arrows denote the operations in the matrix model which correspond to these T-dualities. The matrix model solutions are represented by the non-commutativity parameters

$$
\begin{aligned}
\theta^{i j} & =\left[\hat{x}^{i}, \hat{x}^{j}\right], \\
\tilde{\theta}_{i j} & =\left[\hat{p}_{i}, \hat{p}_{j}\right],
\end{aligned}
$$

whose dependence appears in the parentheses.

Finally, it is worth mentioning the case when one moves directly from the $H$-block to the $R$-block. According to the above rule, the T-duality should be achieved by the operation

$$
M_{H \rightarrow R} \cdot(-1)_{R}^{\hat{c}_{i}}=\left(\begin{array}{cc}
0 & \mathbb{1}_{3} \\
\mathbb{1}_{3} & 0
\end{array}\right),
$$

which is indeed also an element of the T-duality group.

\subsection{Position/Momentum space duality}

The doubled formulation of section 3.1 motivated the introduction of the additional Hermitian matrices $\tilde{\mathcal{X}}^{i}$, which facilitated the implementation of twisted doubled tori in the present framework. However, these matrices should not be really dynamical degrees of 
freedom, since they are not present in Matrix theory ${ }^{6}$. Thus we would like here to restrict the previous formulation to the subsectors which contain only the true dynamical components. This procedure is similar to the so-called "polarizations" of [36].

Let us warm up this discussion with the case of the geometric flux $f$, which was presented both as a twisted torus in section 2.3 and as a twisted doubled torus in section 3.1. As we already mentioned, the overlapping non-trivial compactification conditions and the phase space algebras in these two cases fully coincide, as well as the form of the dynamical matrices $\mathcal{X}_{i}$. This directly shows that there is a trivial projection from one to the other. In a way, the introduction of $\tilde{\mathcal{X}}^{i}$ is totally redundant in this case.

Turning to the $H$-block, which is related to an NS-NS flux background in the supergravity picture, it is again plausible to determine a well-defined projection to a single torus. Indeed, we can consider only the non-trivial conditions

$$
U^{i} \mathcal{X}_{i}\left(U^{i}\right)^{-1}=\mathcal{X}_{i}+1
$$

dressed with the commutation relation

$$
U^{i} U^{j}=e^{-H^{i j k} \hat{\partial}_{k}} U^{j} U^{i}
$$

for the operators $U^{i}$. The conditions (3.49) define a compactification of matrix theory on a torus, while the relation (3.50) indicates that the algebra of functions on this torus is deformed, its non-commutativity being controlled by $H^{i j k}$. We already discussed that this non-commutativity is related to the presence of a non-vanishing and non-constant B-field.

The next and more interesting situation is the $Q$-block. Can we define a projection from the twisted doubled torus to a single toroidal-like compactification? Or, alternatively, can we define a legitimate Matrix theory compactification based on the algebra (3.21)? Suppressing for a moment the $\mathcal{A}_{i}$ part in the connections, let us examine what happens for the standard form of the solutions, i.e. when $\mathcal{X}_{i}=i \hat{\partial}_{i}$ and $U^{i}=e^{i \hat{x}^{i}}$. It is straightforward to obtain

$$
U^{1} \mathcal{X}_{2}\left(U^{1}\right)^{-1}=\mathcal{X}_{2}-\hat{x}^{3} .
$$

This is a bizarre relation which at first sight does not seem to have a clear interpretation apart from the one in the extended context of twisted doubled tori, where the $\hat{x}^{3}$ is actually $\tilde{\mathcal{X}}^{3}$. Furthermore, presently the operators $U^{i}$ commute among themselves, which makes the situation even more obscure.

In order to clarify the above situation let us make the following observations. Define the deformation parameters

$$
\begin{aligned}
\left.\theta^{i j}\right|_{f} & =f^{i j}{ }_{k} \hat{x}^{k}, \\
\left.\tilde{\theta}_{i j}\right|_{Q} & =Q_{i j}{ }^{k} \hat{p}_{k},
\end{aligned}
$$

\footnotetext{
${ }^{6}$ However, note that they could be thought of as coexisting sectors of Matrix theory, i.e. different multibrane solutions which are combined block-diagonally as e.g. in Ref. [12]. Moreover, it is conceivable that $\tilde{\mathcal{X}}^{i}$ may be part of a "doubled matrix model", which could serve as a non-perturbative definition of double field theory [62]. We shall not explore further these possibilities in the present paper.
} 
in obvious notation. There is already a flavour of duality in these relations, which is intensified by looking at the $U / \tilde{U}$ relations:

$$
\begin{aligned}
f: & U^{i} U^{j}\left(U^{i}\right)^{-1}\left(U^{j}\right)^{-1}=f^{i j}{ }_{k}\left(U^{k}\right)^{-1}, & \tilde{U}_{i} \tilde{U}_{j}=\tilde{U}_{j} \tilde{U}_{i}, \\
Q: & \tilde{U}_{i} \tilde{U}_{j}\left(\tilde{U}_{i}\right)^{-1}\left(\tilde{U}_{j}\right)^{-1}=Q_{i j}{ }^{k} \tilde{U}_{k}, & U^{i} U^{j}=U^{j} U^{i},
\end{aligned}
$$

It is then clear that the structure of $U^{i}$ in the $f$-block mimics the structure of $\tilde{U}_{i}$ in the $Q$-block and vice versa. Thus we can argue that the projection which defines a compactification of Matrix theory in the $Q$-block case is the one to the tilded subsector. In particular, we consider the tilded quantities $\tilde{\mathcal{X}}^{i}$ and $\tilde{U}_{i}$ with the conditions

$$
\begin{aligned}
\tilde{U}_{i} \tilde{\mathcal{X}}^{i}\left(\tilde{U}_{i}\right)^{-1} & =\tilde{\mathcal{X}}^{i}+1, \\
\tilde{U}_{2} \tilde{\mathcal{X}}^{1}\left(\tilde{U}_{2}\right)^{-1} & =\tilde{\mathcal{X}}^{1}-\tilde{\mathcal{X}}^{3}, \\
\tilde{U}_{3} \tilde{\mathcal{X}}^{1}\left(\tilde{U}_{3}\right)^{-1} & =\tilde{\mathcal{X}}^{1}+\tilde{\mathcal{X}}^{2} .
\end{aligned}
$$

The dynamical matrices are now the $\tilde{\mathcal{X}}^{i}$ instead. This compactification is now the twisted torus compactification on the dual space to the one operating in the case of the geometric flux $f$. More specifically, let us recall that in the position representation of quantum mechanics the classical variables are mapped to Hermitian operators as

$$
x \rightarrow \hat{x}, \quad p \rightarrow \hat{p}=-i \hbar \frac{\partial}{\partial x}
$$

while in the momentum representation the correspondence is

$$
x \rightarrow \hat{x}=i \hbar \frac{\partial}{\partial p}, \quad p \rightarrow \hat{p} .
$$

This simple observation shows that there is an exact correspondence between geometric $f$ flux in the position space and non-geometric $Q$ flux in the momentum space:

$$
\left.\theta^{i j}\right|_{f} \quad \text { in } \quad \hat{x} \text {-space }\left.\longleftrightarrow \tilde{\theta}_{i j}\right|_{Q} \text { in } \hat{p} \text {-space . }
$$

There is a final case which one would like to project, that of the $R$-block. As expected, this exhibits similar features to the $Q$-block case and it has to be interpreted as above. In particular, assuming the algebra (3.29) with $\mathcal{X}_{i}=i \hat{\partial}_{i}$ and $U^{i}=e^{i \hat{x}^{i}}$, we obtain

$$
\begin{aligned}
U^{i} \mathcal{X}_{i}\left(U^{i}\right)^{-1} & =\mathcal{X}_{i}+1 \\
U^{i} U^{j} & =U^{j} U^{i}
\end{aligned}
$$

This might seem a totally well-defined compactification, i.e. a compactification on a standard torus such as the ones we referred to in section 2.2 with $\theta^{i j}=0$. However, the situation is not as simple as this since it turns out that in the present case the dynamical matrices $\mathcal{X}_{i}$ are non-associative:

$$
\left[\mathcal{X}_{i}, \mathcal{X}_{j}, \mathcal{X}_{k}\right] \neq 0
$$

This is rather expected in view of the known properties of non-geometric compactifications [47-50], but it is awkward to think that a set of Hermitian matrices does not satisfy the Jacobi identity. In fact it is then impossible to represent them on a Hilbert space. The 
discussion of the $Q$-block comes to the rescue, since we can instead consider the projection to the tilded sector, which satisfies the conditions

$$
\tilde{U}_{i} \tilde{\mathcal{X}}^{i}\left(\tilde{U}_{i}\right)^{-1}=\tilde{\mathcal{X}}^{i}+1
$$

with

$$
\tilde{U}_{i} \tilde{U}_{j}=e^{R_{i j k} \hat{x}^{k}} \tilde{U}_{j} \tilde{U}_{i}
$$

This comes on a dual footing to the $H$-block case. In particular, the relevant deformation parameters are

$$
\begin{aligned}
\left.\theta^{i j}\right|_{H} & =H^{i j k} \hat{p}_{k}, \\
\left.\tilde{\theta}_{i j}\right|_{R} & =R_{i j k} \hat{x}^{k} .
\end{aligned}
$$

Thus there is an exact correspondence between NS-NS $H$ flux in the position space and non-geometric $R$ flux in the momentum space:

$$
\left.\theta^{i j}\right|_{H} \quad \text { in } \hat{x} \text {-space }\left.\longleftrightarrow \tilde{\theta}_{i j}\right|_{R} \text { in } \hat{p} \text {-space }
$$

In the momentum space the compactification related to the $R$-block is well-defined and moreover the dynamical matrices are associative.

Summarizing,

- In position space, there are well-defined compactifications of Matrix theory with nonconstant non-commutativity $\theta^{i j}$ among the coordinate operators, which is related to the presence of geometric or NS-NS fluxes in the corresponding supergravity compactifications. The compactifications related to the $Q$ - and $R$-algebras which exhibit non-commutativity among the momentum operators are not well-defined.

- In momentum space, the compactifications with non-commutativity $\tilde{\theta}_{i j}$ among the momentum operators are well-defined and they correspond to supergravity with $Q$ and $R$ fluxes. The cases based on the $H$ - and $f$-algebras are presently not well-defined.

The situation we just described has a very similar incarnation in low-energy supergravity. The authors of Refs. [38,39] studied non-geometric compactifications from the perspective of generalized geometry $[63,64]$. They showed that while non-geometric configurations are ill-defined in a frame where the generalized metric is parametrized by the B-field $B_{i j}$, they become well-defined in another frame where the generalized metric is parametrized by the antisymmetric bivector $\beta^{i j}$ of generalized geometry. In the second frame, geometric configurations are instead ill-defined. This means that one has to choose the appropriate generalized vielbein which would yield the correct, i.e. well-defined, Lagrangian for each configuration.

The above discussion allows us to make the reasonable speculation that just as the deformation parameter $\theta$ is related to the B-field via

$$
\theta^{i j} \sim\left(B_{i j}\right)^{-1},
$$


there should exist a similar dependence among the deformation parameter $\tilde{\theta}$ and the bivector $\beta$ of generalized geometry as

$$
\tilde{\theta}_{i j} \sim\left(\beta^{i j}\right)^{-1}
$$

The latter relation should be studied in detail by comparison of the corresponding effective actions.

Moreover, let us remind that in supergravity $f$ is a (spin) connection and $H$ is a tensor field. The above duality between position and momentum space in the matrix model indicates that in momentum space $Q$ should instead be a connection and $R$ should be a tensor field. A similar observation was made in [39].

A final remark regards the index structure of the encountered quantities. Observe that the indices in the T-duality chain (1.1) are in exactly the opposite position to the corresponding parameters in the phase space algebras. Recall that the former are related to the supergravity picture while the latter to the matrix model picture. Then the relations (3.66) and (3.67) explain the above index structure.

\subsection{Resolution of non-associativity and flux quantization}

Let us now make some important remarks regarding the non-assocativity we encountered above. First of all, we could say that there are two types of non-associativity which have to be treated differently. The first type is the one among the dynamical degrees of freedom $\mathcal{X}_{i}$. This issue was already discussed in the previous subsection within position-momentum space duality. We saw that in the appropriate representation, where the compactification is well-defined, there is no sign of non-associativity. In particular, for the $R$-type solution in momentum space the dynamical degrees of freedom are perfectly associative.

However, there is a second type of non-associativity which we have not discussed in detail so far. This has a common origin to the first one, i.e. the phase space algebra, but it regards the non-dynamical algebraic elements $U^{i}$. Indeed, let us look at the $H$-block, with algebra (3.12) and commutation relation among the $U^{i}$ as in Eq. (3.50). It is strightforward to compute that

$$
U^{i}\left(U^{j} U^{k}\right)=e^{\frac{i}{2} H^{i j k}}\left(U^{i} U^{j}\right) U^{k} .
$$

This relation should be thought of as an anomalous 3-cocycle. Indeed, exactly the same relation may be found in [54], where its appearance in a physical system is discussed. However, such a 3-cocycle cannot be tolerated. In the words of Jackiw, "It is important to appreciate that non-associating quantities cannot be represented by well-defined linear operators, acting on a vector or Hilbert space, since by definition operators on vectors necessarily associate" (see page 19 of Ref. [54]). The resolution of this problem leads naturally to a Dirac quantization condition. Requiring associativity to be restored in Eq. (3.68) we directly obtain

$$
H^{i j k}=4 \pi n, \quad n \in \mathbb{Z} .
$$

Therefore we observe that the analog of the $H$ flux in the framework of Matrix theory has to obey a quantization condition. This is very plausible because in string theory fluxes have to be quantized [2]. 
Let us move on to the geometric flux and examine what happens in this case. Taking into account the algebra (3.5) and the commutation relation among the operators $U^{i}$, we obtain

$$
U^{i}\left(U^{j} U^{k}\right)=\left(U^{i} U^{j}\right) U^{k}
$$

namely the present situation is already associative. This is expected, since the $f$ flux does not arise from a $p$-form source, but it is a metric flux.

As far as the $Q$ and $R$ cases are concerned, there is not much to add. As we already discussed above, they provide well-defined solutions in the momentum space, where they play the corresponding role of $f$ and $H$. Therefore, $R$ obeys a quantization condition much like $H$, while for $Q$ the corresponding operators already associate.

It should be noted that apart from the above operators, associativity should be guaranteed for the gauge fields $\mathcal{A}$ of each solution. These are functions of the hatted operators $\hat{U}^{i}$, which associate whenever the unhatted ones do. Therefore, the above discussion applies equally well for the gauge fields too.

Let us mention that in the recent Ref. [65] and in the context of double field theory it is found that large gauge transformations do associate even in cases when the coordinate maps do not. It is notable that this exhibits a similarity to our present discussion in a rather different framework.

\subsection{Putting the blocks together}

It is well-known that in supergravity it is possible to consider compactifications where different types of fluxes coexist ${ }^{7}$. For example one can consider simultaneously geometric and NS-NS fluxes, i.e. a twisted torus penetrated by an $H$ flux. Therefore it is reasonable to ask whether the different algebraic blocks described above may be combined to yield Matrix theory compactifications with a superimposition of deformation parameters $\theta$ and/or $\tilde{\theta}$. This question boils down to the attempt of defining and solving the appropriate compactification conditions which would yield the general phase space algebra

$$
\begin{aligned}
& {\left[\hat{x}^{i}, \hat{x}^{j}\right]=i f^{i j}{ }_{k} \hat{x}^{k}+H^{i j k} \hat{\partial}_{k},} \\
& {\left[\hat{\partial}_{i}, \hat{\partial}_{j}\right]=Q_{i j}{ }^{k} \hat{\partial}_{k}-i R_{i j k} \hat{x}^{k},} \\
& {\left[\hat{\partial}_{i}, \hat{x}^{j}\right]=\delta_{i}^{j}-Q_{i k}{ }^{j} \hat{x}^{k}-i f^{j k} \hat{\partial}_{k} .}
\end{aligned}
$$

In the doubled formalism of section 3.1, the form of $\mathcal{X}_{i}, U^{i}$ and the corresponding tilded ones leads to some compactification conditions which combine all the blocks that we discussed in section 3.1. Although these conditions define some non-commutative twisted doubled torus, for Matrix theory we would like to have a projection on a single set of dynamical variables (see, however, footnote 3). Let us now discuss some of them.

A first possibility is to consider $\mathcal{X}_{i}$ as the Matrix theory variables. This choice includes the first two projections of section 3.3 but it also allows for the combination of the two. This

\footnotetext{
${ }^{7}$ Usually this is indispensable in order to obtain a true string vacuum solving the string equations of motion, which is not the case for the toy model of a twisted 3-torus. R-R fluxes are then important too, but we will not discuss them here.
} 
amounts to the deformation parameters

$$
\theta^{i j}=H^{i j k} \hat{p}_{k}+f_{k}^{i j} \hat{x}^{k}, \quad \tilde{\theta}_{i j}=0 .
$$

The first term in $\theta$ corresponds to a non-constant B-field, while the second term to a geometric flux. Thus, this situation would be related to a twisted torus with NS-NS flux in supergravity.

Equally well we could project to the $\tilde{X}^{i}$ sector, which would now provide the dynamical variables of Matrix theory. This choice includes the latter two projections of section 3.3, as well as their combination. As discussed above, both these projections are ill-defined in $\hat{x}$-space but they become well-defined in $\hat{p}$-space. The deformation parameters are now

$$
\theta^{i j}=0, \quad \tilde{\theta}_{i j}=Q_{i j}{ }^{k} \hat{p}_{k}+R_{i j k} \hat{x}^{k}
$$

and in $\hat{p}$-space they define a Matrix theory compactification analogous to a supergravity background with $Q$ and $R$ fluxes.

It is not clear how one could combine all the fluxes in a single case. One possibility would be to consider the sum of two twisted tori, $\tilde{\mathrm{T}}_{A}^{3} \oplus \tilde{\mathrm{T}}_{B}^{3}$ and associate the Hermitian matrices $\mathcal{X}_{i}$ to the first twisted torus and the matrices $\mathcal{X}_{i+3}$ to the second one, for $i=1,2,3$. Then one could think of using the solution related to the Eq. (3.72) on the first torus and the solution associated to Eq. (3.73) on the second. This is a legitimate possibility but its interpretation is rather obscure and therefore we would not like to pursue it further in the present paper.

\section{Remarks on the gauge theory}

In the previous section we determined several types of solutions of Matrix theory compactified on deformed tori. In the prototype example of a torus with constant noncommutativity, when the solution is substituted back into the original action functional the effective action describes a non-commutative gauge theory coupled to scalars and fermions [16]. This resulting action was subsequently compared to the action of a string moving in the background of a constant B-field. This comparison further supports the correspondence between non-commutativity parameters and background field values [66-68].

Generically, the compactification of the matrix model on a standard 3-torus will lead to the following tree-level effective action:

$$
\mathcal{S}_{\text {eff }} \propto \int d t \operatorname{Tr}\left\{-\dot{\mathcal{D}}_{i}^{2}+\dot{\mathcal{A}}_{m}^{2}+\frac{1}{2}\left[\mathcal{D}_{i}, \mathcal{D}_{j}\right]^{2}-i\left[\mathcal{D}_{i}, \mathcal{A}_{m}\right]^{2}-\frac{1}{2}\left[\mathcal{A}_{m}, \mathcal{A}_{n}\right]^{2}+\text { fermions }\right\} .
$$

Here we replaced the matrices $\mathcal{X}_{i}$ with their solution, namely with covariant derivatives. Ignoring the scalar part of the action coming from $\mathcal{X}_{m}$ and defining $F_{i j}=\left[\mathcal{D}_{i}, \mathcal{D}_{j}\right]$ one obtains

$$
\mathcal{S} \propto \int d t \operatorname{Tr}\left(F_{i j} F^{i j}\right) .
$$


In the case of compactification on a non-commutative torus [16], the action (4.1) is defined on the dual non-commutative torus given by the relations (2.8). It is possible to represent the action (4.1) on the space of commuting variables and rewrite the trace over infinitedimensional matrices as

$$
\operatorname{Tr} \rightarrow \int d^{3} x \operatorname{tr}
$$

where $x^{i}$ are periodic coordinates on $\mathrm{T}^{3}$ and tr denotes the trace over n-dimensional Hermitean matrices $\mathcal{A}_{i}$. Representing the matrices $\mathcal{A}_{i}$ as functions of commuting variables $A_{i}\left(x^{j}\right)$ one obtains the non-commutative field strength in the form

$$
F_{i j}=\partial_{i} A_{j}-\partial_{j} A_{i}+i A_{i} \star A_{j}-i A_{j} \star A_{i},
$$

where the Moyal-Weyl $\star$ product

$$
f \star g=\left.e^{\frac{i}{2} \frac{\partial}{\partial x^{i}} \hat{\theta}^{i j} \frac{\partial}{\partial y^{j}}} f(x) g(y)\right|_{y \rightarrow x},
$$

encodes the non-commutativity of the algebra of functions (2.8) on the non-commutative torus.

\subsection{Gauge theory with fluxes}

Let us now discuss some features of the gauge theory resulting from the compactifications with fluxes. In the following we concentrate on the pure gauge sector of the compactified action.

In the case of geometric flux, the solutions of the compactification conditions are:

$$
\begin{aligned}
\mathcal{X}_{i}=i \mathcal{D}_{i} & =i \hat{\partial}_{i}+\mathcal{A}_{i}(\hat{U})+i f_{i}{ }^{j k} \mathcal{A}_{j}(\hat{U}) \hat{\partial}_{k}, \\
\tilde{\mathcal{X}}^{i} & =i \tilde{\mathcal{D}}^{i}=(-1)^{c_{i}} x^{i}+\tilde{\mathcal{A}}^{i}(\tilde{U})-f_{j}{ }^{i k} \mathcal{A}_{k}(\hat{U}) x^{j}
\end{aligned}
$$

Note that $\tilde{\mathcal{A}}^{i}$ are polynomial functions of $\tilde{U}_{k}=\exp \left((-)^{c_{k}} \partial_{k}\right)$, while $\mathcal{A}_{i}$ depend on $\hat{U}^{j}=\exp \left(i \hat{y}^{j}\right)$, with $\hat{y}^{j}=\hat{x}^{j}-i f_{k}^{j i} \hat{x}^{k} \hat{\partial}_{i}$. The gauge transformations corresponding to the compactification of the original action on the twisted doubled torus are generated by $U^{i}$ :

$$
\begin{aligned}
& U^{i} \hat{\mathcal{A}}_{j}\left(U^{i}\right)^{-1}=\hat{\mathcal{A}}_{j}-f_{j}{ }^{i k} \hat{\mathcal{A}}_{k}, \\
& U^{i} \tilde{\mathcal{A}}^{j}\left(U^{i}\right)^{-1}=\tilde{\mathcal{A}}^{j}+f_{k}{ }^{i j} \tilde{\mathcal{A}}^{k},
\end{aligned}
$$

and $\tilde{U}_{i}$

$$
\begin{aligned}
\tilde{U}_{i} \hat{\mathcal{A}}_{j}\left(\tilde{U}_{i}\right)^{-1} & =\hat{\mathcal{A}}_{j}, \\
\tilde{U}_{i} \tilde{\mathcal{A}}^{j}\left(\tilde{U}_{i}\right)^{-1} & =\tilde{\mathcal{A}}^{j}
\end{aligned}
$$

Let us recall that the corrected gauge field has the form $\hat{\mathcal{A}}_{i}=\mathcal{A}_{i}+i f_{i}{ }^{j k} \mathcal{A}_{j} \hat{\partial}_{k}$. The algebra of these gauge generators, given in Eq. (3.7), represents in the matrix model framework the gauge algebra of the corresponding string compactification, which was determined in Ref. [30]. 
Inserting the solutions (4.5) back into the original action (2.1) leads to the following effective action:

$$
\mathcal{S}_{\text {eff }} \propto \int d t \operatorname{Tr}\left(\left[\mathcal{D}_{i}, \mathcal{D}_{j}\right]^{2}+2\left[\mathcal{D}_{i}, \tilde{\mathcal{D}}^{j}\right]^{2}+\left[\tilde{\mathcal{D}}^{i}, \tilde{\mathcal{D}}^{j}\right]^{2}\right)
$$

As we noticed before, the dual formulation is redundant in the case of geometric flux. The gauge (sub)algebra generated by $U^{i}$ closes, so projecting on the physical torus we get

$$
\mathcal{S} \propto \int d t \operatorname{Tr}\left[\mathcal{D}_{i}, \mathcal{D}_{j}\right]^{2}=\int d t \operatorname{Tr}\left(F_{i j} F^{i j}\right)
$$

where the non-commutative field strength in the present case is defined in terms of $\hat{\mathcal{A}}_{i}$ as $F_{i j}=\partial_{i} \hat{\mathcal{A}}_{j}-\partial_{j} \hat{\mathcal{A}}_{i}-i\left[\hat{\mathcal{A}}_{i}, \hat{\mathcal{A}}_{j}\right]$.

Notice that the operators $\hat{U}^{j}$ generate the gauge transformations in the resulting gauge theory (4.9). Moreover, one can show that these operators define the compactification on the dual torus:

$$
\begin{aligned}
\hat{U}^{i} \mathcal{X}_{i}\left(\hat{U}^{i}\right)^{-1} & =\mathcal{X}_{i}+1, \\
\hat{U}^{1} \mathcal{X}_{3}\left(\hat{U}^{1}\right)^{-1} & =\mathcal{X}_{3}-2 \mathcal{X}_{2}, \\
\hat{U}^{2} \mathcal{X}_{3}\left(\hat{U}^{2}\right)^{-1} & =\mathcal{X}_{3}+2 \mathcal{X}_{1},
\end{aligned}
$$

with

$$
\hat{U}^{i} \hat{\mathcal{A}}_{j}\left(\hat{U}^{i}\right)^{-1}=\hat{\mathcal{A}}_{j}
$$

This supports our claim that the non-constant non-commutativity of coordinate operators corresponds to turning on a geometric flux in supergravity.

Representing the action (4.9) on the space of commuting coordinates is a non-trivial task. However, it is important to notice that the non-commutative structure of the algebra of functions on the torus would be encoded using the $\star$ product:

$$
f \star g=\left.e^{-\frac{i}{2} f_{k}^{i j} x^{k} \frac{\partial}{\partial y^{i}} \frac{\partial}{\partial z^{j}}} f(y) g(z)\right|_{y, z \rightarrow x},
$$

which is associative, as is the whole algebra of functions on the twisted torus.

Let us turn to the compactification incorporating the $H$ flux. This case resulted in the following solutions:

$$
\begin{aligned}
\mathcal{X}_{i}=i \mathcal{D}_{i} & =i \hat{\partial}_{i}+\mathcal{A}_{i}(\hat{U}) \\
\tilde{\mathcal{X}}^{i}=i \tilde{\mathcal{D}}^{i} & =x^{i}+\tilde{\mathcal{A}}^{i}(\tilde{U})+i H^{i j k} \mathcal{A}_{j}(\hat{U}) \partial_{k}
\end{aligned}
$$

The gauge transformations are generated by $U^{i}$ and $\tilde{U}_{i}$ :

$$
\begin{aligned}
& U^{i} \mathcal{A}_{j}\left(U^{i}\right)^{-1}=\mathcal{A}_{j}, \quad U^{i} \hat{\tilde{\mathcal{A}}}^{j}\left(U^{i}\right)^{-1}=\hat{\tilde{\mathcal{A}}}^{j}+H^{i j k} \mathcal{A}_{k}, \\
& \tilde{U}_{i} \mathcal{A}_{j}\left(\tilde{U}_{i}\right)^{-1}=\mathcal{A}_{j}, \quad \tilde{U}_{i} \hat{\tilde{\mathcal{A}}}^{j}\left(\tilde{U}_{i}\right)^{-1}=\hat{\tilde{\mathcal{A}}}^{j}
\end{aligned}
$$

The gauge algebra, given in (3.14), is now more involved. Unlike the case of geometric flux, here the algebra of generators $U^{i}$ does not close. Inserting the solutions (4.13) in the 
original BFSS action we again obtain (4.8), but in this case the second term under the integral will have a contribution to the projected action. We find that the projected action on the (plain) torus is of the form:

$$
\mathcal{S} \propto \int d t \operatorname{Tr}\left(\frac{1}{4} F_{i j}^{2}+\frac{1}{2} H^{i j k} \mathcal{X}_{k} F_{i j}-H^{i j k} \mathcal{A}_{k} F_{i j}+\frac{i}{2} H^{i j k}\left[\mathcal{A}_{i}, \mathcal{A}_{j}\right] \mathcal{X}_{k}+\mathcal{O}\left(H^{2}\right)\right) .
$$

The first H-dependent term is basically a Myers term [69], here obtained from the fluctuations without a need to modify the original action. Let us note that such a term was also obtained in Refs. [70,71] from the expansion of the Dirac-Born-Infeld action. The second and the third H-dependent terms appear as (parts of) the Chern-Simons action. Let us recall that here we substituted the solutions in the tree-level matrix model action. It would be very interesting to determine the 1-loop effective action of the solutions we discussed and analyze the corresponding new terms. This task may be pursued along the lines of techniques used in Refs. [72,73].

Moreover, the gauge tranformations induced by $\hat{U}^{i}$ define a compactification on the dual torus:

$$
\hat{U}^{i} \mathcal{X}_{j}\left(\hat{U}^{i}\right)^{-1}=\mathcal{X}_{j}+1, \quad \hat{U}^{i} \hat{\mathcal{A}}_{j}\left(\hat{U}^{i}\right)^{-1}=\hat{\mathcal{A}}_{j},
$$

showing that this matrix model compactification is related to a supergravity compactification on a torus with NS-NS flux.

The compactifications incorporating $Q$ and $R$ fluxes do not reveal any new structure. Defining the physical space as the one where the algebra of functions is associative, lead us to the projection on the dual momentum space. The effective tree-level gauge action in these cases will be dual to the ones for $f$ and $H$ cases as discussed in section 3.3.

\section{Conclusions}

The BFSS model or Matrix theory is a matrix model which, in its $N \rightarrow \infty$ limit, is conjectured to be equivalent to uncompactified M theory [1]. Thus, if Matrix theory indeed serves as a non-perturbative definition of $\mathrm{M}$ theory, it should contain its low-energy limit, namely 11-dimensional supergravity on flat spacetime. Moreover, compactifications of the matrix model should provide another description of compactified supergravity backgrounds. Progress towards this direction revealed that matrix compactifications on non-commutative tori are related to supergravity compactifications with background fields $[16,66]$.

In the present paper we explored connections between compactifications of Matrix theory and flux compactifications of supergravity, with geometric, NS-NS and non-geometric fluxes. The quantum-mechanical nature of the BFSS model assigns an important role to the phase space. In particular, different non-commutative deformations of the phase space

algebra lead to certain solutions of the model and the parameters of the non-commutativity can be related to fluxes in the corresponding supergravity compactification. Moreover, the algebraic building blocks for these solutions can be related via certain operations which provide a matrix model realization of the T-duality chain

$$
H_{i j k} \stackrel{T_{k}}{\longrightarrow} f_{i j}{ }^{k} \stackrel{T_{j}}{\longrightarrow} Q_{i}^{j k} \stackrel{T_{i}}{\longrightarrow} R^{i j k}
$$


The T-duality pattern results from canonical transformations exchanging position and momentum operators in the phase space.

The role of the phase space in this framework becomes even more central under the realization that certain non-associative structures emerge when the compactification conditions are solved. Essentially there are two types of such structures: (a) non-associativity of the dynamical degrees of freedom of the theory, i.e. the Hermitian matrices and (b) nonassociativity between gauge transformations represented by unitary operators. They both have a common origin in the non-associativity of the phase space algebra. However, since the above quantities should be operators on a Hilbert space, their non-associativity has to be resolved $[53,54]$.

The resolution of non-associativity in the above two cases follows a different path. In the case of the dynamical degrees of freedom, where non-associativity appears only in the solution associated to a non-geometric $R$ flux, the interpretation is based on a duality between position space and momentum space. Thus, a solution which is not well-defined in position space turns out to be perfectly well-defined in momentum space. A similar argument holds for the $Q$ flux case. Thus, while the solutions related to geometric and NSNS fluxes are defined in position space, the ones for the non-geometric fluxes are defined on momentum space. What is more, in momentum space a $Q$ flux plays the role of a geometric flux (i.e. a connection in supergravity language), while the $R$ flux plays the role of the $H$ flux (i.e. a tensor field in supergravity). The latter observation indicates that much like the non-commutativity of coordinates exhibits a reciprocal relation to the B-field, the noncommutativity of momenta should be reciprocally related to the bivector $\beta$ of generalized geometry.

As for the translation operators, the requirement of associativity leads to a quantization condition for the flux. Therefore, fluxes in compactified Matrix theory appear to be quantized due to this requirement. This is a very welcome feature of the matrix model, since in a quantum theory charges have to be quantized anyway.

Finally, we discussed some aspects of the effective gauge theory obtained from these compactifications. The transformations of the gauge fields were provided and the effective action obtained by inserting the solutions back into the tree-level action of the matrix model was determined. It is notable that in the NS-NS flux case we obtained terms which are related to the Myers and Chern-Simons terms. An interesting next step would be to calculate the 1-loop effective action and try to compare it with the Dirac-Born-Infeld and Chern-Simons actions.

Acknowledgements. We would like to thank Ricardo Schiappa and Huyn Seok Yang for useful discussions. Heartfelt thanks go to Nikos Prezas for numerous discussions on topics related to the present work over the previous years. This work was partially supported by the SFB-Transregio TR33 "The Dark Universe" (Deutsche Forschungsgemeinschaft), the European Union 7th network program "Unification in the LHC era" (PITN-GA-2009237920) and the Alexander von Humboldt foundation. A. C. is grateful to the Simons Centre for Geometry and Physics and the organizers of the Simons Summer Workshop in Mathematics and Physics 2012 for warm hospitality and a stimulating working atmosphere. 


\section{References}

[1] T. Banks, W. Fischler, S. H. Shenker and L. Susskind, Phys. Rev. D55 (1997) 51125128 [hep-th/9610043].

[2] M. Grana, Phys. Rept. 423 (2006) 91 [hep-th/0509003].

[3] M. R. Douglas and S. Kachru, Rev. Mod. Phys. 79 (2007) 733 [hep-th/0610102].

[4] T. Eguchi and H. Kawai, Phys. Rev. Lett. 48 (1982) 1063.

[5] N. Ishibashi, H. Kawai, Y. Kitazawa and A. Tsuchiya, Nucl. Phys. B 498 (1997) 467 [arXiv:hep-th/9612115].

[6] H. Aoki, N. Ishibashi, S. Iso, H. Kawai, Y. Kitazawa and T. Tada, Nucl. Phys. B 565 (2000) 176 [hep-th/9908141].

[7] H. Aoki, S. Iso, H. Kawai, Y. Kitazawa and T. Tada, Prog. Theor. Phys. 99 (1998) 713 [hep-th/9802085].

[8] J. Nishimura and F. Sugino, JHEP 0205 (2002) 001 [hep-th/0111102].

[9] H. Kawai, S. Kawamoto, T. Kuroki, T. Matsuo and S. Shinohara, Nucl. Phys. B 647 (2002) 153 [hep-th/0204240].

[10] H. Steinacker, Class. Quant. Grav. 27 (2010) 133001 [arXiv:1003.4134 [hep-th]].

[11] H. Aoki, Prog. Theor. Phys. 125 (2011) 521 [arXiv:1011.1015 [hep-th]].

[12] A. Chatzistavrakidis, H. Steinacker and G. Zoupanos, JHEP 1109 (2011) 115 [arXiv:1107.0265 [hep-th]].

[13] S. -W. Kim, J. Nishimura and A. Tsuchiya, Phys. Rev. Lett. 108 (2012) 011601 [arXiv:1108.1540 [hep-th]].

[14] S. -W. Kim, J. Nishimura and A. Tsuchiya, arXiv:1110.4803 [hep-th].

[15] J. Nishimura, arXiv:1205.6870 [hep-lat].

[16] A. Connes, M. R. Douglas and A. S. Schwarz, JHEP 9802 (1998) 003 [hep-th/9711162].

[17] D. A. Lowe, H. Nastase and S. Ramgoolam, Nucl. Phys. B 667 (2003) 55 [hep-th/0303173].

[18] A. Chatzistavrakidis and L. Jonke, Phys. Rev. D 85 (2012) 106013 [arXiv:1202.4310 [hep-th]].

[19] M. Anazawa, Nucl. Phys. B 569 (2000) 680 [hep-th/9905055].

[20] S. Hellerman, J. McGreevy and B. Williams, JHEP 0401 (2004) 024 [hep-th/0208174]. 
[21] A. Dabholkar and C. Hull, JHEP 0309 (2003) 054 [hep-th/0210209].

[22] A. Flournoy, B. Wecht and B. Williams, Nucl. Phys. B 706 (2005) 127 [hep-th/0404217].

[23] O. J. Ganor, S. Ramgoolam and W. Taylor, Nucl. Phys. B 492 (1997) 191 [hep-th/9611202].

[24] D. Brace, B. Morariu and B. Zumino, Nucl. Phys. B 545 (1999) 192 [hep-th/9810099].

[25] D. Brace, B. Morariu and B. Zumino, Nucl. Phys. B 549 (1999) 181 [hep-th/9811213].

[26] J. Shelton, W. Taylor and B. Wecht, JHEP 0510 (2005) 085 [hep-th/0508133].

[27] J. Shelton, W. Taylor and B. Wecht, JHEP 0702 (2007) 095 [hep-th/0607015].

[28] S. Kachru, M. B. Schulz, P. K. Tripathy and S. P. Trivedi, JHEP 0303 (2003) 061 [hep-th/0211182].

[29] J. Scherk and J. H. Schwarz, Nucl. Phys. B 153 (1979) 61.

[30] N. Kaloper and R. C. Myers, JHEP 9905 (1999) 010 [hep-th/9901045].

[31] C. M. Hull and R. A. Reid-Edwards, Fortsch. Phys. 57 (2009) 862 [hep-th/0503114].

[32] G. Dall'Agata and N. Prezas, JHEP 0510 (2005) 103 [hep-th/0509052].

[33] C. M. Hull and R. A. Reid-Edwards, JHEP 0610 (2006) 086 [hep-th/0603094].

[34] M. Grana, R. Minasian, M. Petrini and A. Tomasiello, JHEP 0705 (2007) 031 [hep-th/0609124].

[35] C. M. Hull, JHEP 0510 (2005) 065 [hep-th/0406102].

[36] C. M. Hull and R. A. Reid-Edwards, JHEP 0909 (2009) 014 [arXiv:0902.4032 [hep-th]].

[37] G. Dall'Agata, N. Prezas, H. Samtleben and M. Trigiante, Nucl. Phys. B 799 (2008) 80 [arXiv:0712.1026 [hep-th]].

[38] D. Andriot, M. Larfors, D. Lüst and P. Patalong, JHEP 1109 (2011) 134 [arXiv:1106.4015 [hep-th]].

[39] D. Andriot, O. Hohm, M. Larfors, D. Lüst and P. Patalong, Phys. Rev. Lett. 108 (2012) 261602 [arXiv:1202.3060 [hep-th]].

[40] G. Aldazabal, W. Baron, D. Marques and C. Nunez, JHEP 1111 (2011) 052 [Erratumibid. 1111 (2011) 109] [arXiv:1109.0290 [hep-th]].

[41] D. Geissbuhler, JHEP 1111 (2011) 116 [arXiv:1109.4280 [hep-th]].

[42] M. Grana and D. Marques, JHEP 1204 (2012) 020 [arXiv:1201.2924 [hep-th]]. 
[43] G. Dibitetto, J. J. Fernandez-Melgarejo, D. Marques and D. Roest, arXiv:1203.6562 [hep-th].

[44] R. Blumenhagen, A. Deser, D. Lüst, E. Plauschinn and F. Rennecke, J. Phys. A 44 (2011) 385401 [arXiv:1106.0316 [hep-th]].

[45] R. Blumenhagen, A. Deser, E. Plauschinn and F. Rennecke, arXiv:1205.1522 [hep-th].

[46] D. S. Berman, E. T. Musaev and D. C. Thompson, arXiv:1208.0020 [hep-th].

[47] V. Mathai and J. M. Rosenberg, Commun. Math. Phys. 253 (2004) 705 [hep-th/0401168].

[48] P. Bouwknegt, K. Hannabuss and V. Mathai, Commun. Math. Phys. 264 (2006) 41 [hep-th/0412092].

[49] I. Ellwood and A. Hashimoto, JHEP 0612 (2006) 025 [hep-th/0607135].

[50] P. Grange and S. Schäfer-Nameki, Nucl. Phys. B 770 (2007) 123 [hep-th/0609084].

[51] D. Lüst, JHEP 1012 (2010) 084 [arXiv:1010.1361 [hep-th]].

[52] D. Lüst, arXiv:1205.0100 [hep-th].

[53] R. Jackiw, Phys. Rev. Lett. 54 (1985) 159.

[54] R. Jackiw, MIT-CTP-1298; HIGH ENERGY PHYSICS 1985: proceedings of the Yale [Second] Theoretical Advanced Study Institute. Edited by Mark J. Boswick and Feza Gursey. Singapore, World Scientific, 1985.

[55] R. J. Szabo, Phys. Rept. 378 (2003) 207 [hep-th/0109162].

[56] D. Mylonas, P. Schupp and R. J. Szabo, arXiv:1207.0926 [hep-th].

[57] R. Blumenhagen and E. Plauschinn, J. Phys. A 44 (2011) 015401 [arXiv:1010.1263 [hep-th]].

[58] C. Condeescu, I. Florakis and D. Lust, JHEP 1204 (2012) 121 [arXiv:1202.6366 [hep-th]].

[59] P. -M. Ho, Phys. Lett. B 434 (1998) 41 [hep-th/9803166].

[60] W. Taylor, Phys. Lett. B 394 (1997) 283 [hep-th/9611042].

[61] A. Chatzistavrakidis, Phys. Rev. D 84 (2011) 106010 [arXiv:1108.1107 [hep-th]].

[62] C. Hull and B. Zwiebach, JHEP 0909 (2009) 099 [arXiv:0904.4664 [hep-th]].

[63] N. Hitchin, Quart. J. Math. Oxford Ser. 54 (2003) 281 [math/0209099 [math-dg]].

[64] M. Gualtieri, math/0401221 [math-dg].

[65] O. Hohm and B. Zwiebach, arXiv:1207.4198 [hep-th]. 
[66] M. R. Douglas and C. M. Hull, JHEP 9802 (1998) 008 [hep-th/9711165].

[67] T. Kawano and K. Okuyama, Phys. Lett. B 433 (1998) 29 [hep-th/9803044].

[68] C. -S. Chu and P. -M. Ho, Nucl. Phys. B 550 (1999) 151 [hep-th/9812219].

[69] R. C. Myers, JHEP 9912 (1999) 022 [hep-th/9910053].

[70] L. Cornalba and R. Schiappa, Commun. Math. Phys. 225 (2002) 33 [hep-th/0101219].

[71] P. Bordalo, L. Cornalba and R. Schiappa, Nucl. Phys. B 710 (2005) 189 [hep-th/0409017].

[72] D. N. Blaschke and H. Steinacker, JHEP 1110 (2011) 120 [arXiv:1109.3097 [hep-th]].

[73] Y. Asano, H. Kawai and A. Tsuchiya, Int. J. Mod. Phys. A 27 (2012) 1250089 [arXiv:1205.1468 [hep-th]]. 